\title{
INVESTIGATION OF RUNNING DEPTH STABILITY IN SOIL OF FRONTALLY INSTALLED PLOUGH OF PLOUGHING AGGREGATE ASSEMBLED ACCORDING TO "PUSH-PULL" SCHEME
}

\author{
Volodymyr Bulgakov ${ }^{1}$, Semjons Ivanovs ${ }^{2}$, Hryhorij Kaletnik ${ }^{3}$, Volodymyr Kuvachov ${ }^{4}$ \\ ${ }^{1}$ National University of Life and Environmental Sciences of Ukraine, Ukraine; \\ ${ }^{2}$ Latvia University of Life Sciences and Technologies, Latvia; \\ ${ }^{3}$ Vinnytsia National Agrarian University, Ukraine; \\ ${ }^{4}$ Tavria State Agrotechnological University, Ukraine \\ semjons@apollo.lv
}

\begin{abstract}
To reduce energy consumption in ploughing, the tractor within the aggregate with a frontal plough should have a narrow gauge and high draft and linkage properties. It is suggested to increase the latter ones not by ballasting the tractor or changing the parameters of its under-carriage system, but by using both the mass of the frontally mounted (frontal) plough and the vertical component of its draught resistance. Synthesis of the scheme and justification of the parameters of the ploughing aggregate were carried out by simulating the conditions of its operation on the PC. Two variants of attachment the frontal plough to the frontal linkage mechanism of the tractor were regarded during the research: the first one is characterised by the fact that the inclination angle of the central drawbar of the frontal linkage is less than the inclination angle of the lower links; in the second variant - on the contrary. For a stable movement of the frontal plough along the depth of ploughing with minimal waste of energy for rolling its supporting wheel and insignificant deformation of the soil, the vertical reaction upon the supporting wheel should be, on the one hand, greater than zero, but, on the other hand, close to it. When the frontal plough is pivotally attached to the frontal linkage mechanism of the tractor with the inclination angle of the central drawbar larger than the inclination angle of the lower links, the vertical load on the supporting wheel of the ploughing implement is greater. In contrast to the setting variant of the frontal linkage mechanism of the tractor, when the inclination angle of the central drawbar is less than the inclination angle of the lower links, the vertical load on the supporting wheel of the ploughing implement is 5.5 times larger. The maximum values of the reaction on the supporting wheel are achieved at various inclination angles of the central drawbar and the lower links of the frontal linkage mechanism of the tractor.
\end{abstract}

Keywords: plough, aggregate, depth, stability, movement.

\section{Introduction}

Despite the fact that the system that gains increasing popularity among the technological processes of basic tillage is the "No-till" system, ploughing continues to occupy a prominent place. At the same time ploughing remains one of the most energy-intensive operations of agricultural production $[1 ; 2]$. The increase in traction characteristics of the tractor unit is possible due to an increase in its coupling weight. To reduce energy consumption in ploughing, the tractor within the aggregate with a plough should have a narrow gauge and high draft and linkage properties. Besides, it is suggested to increase the latter ones not by ballasting the tractor or changing the parameters of its under-carriage system, but by using both the mass of the frontally mounted (frontal) plough and the vertical component of its draught resistance [3; 4]. It is assumed that with such an aggregation the trailing mass of the tractor should increase, while skidding of the propulsors and the specific fuel consumption should decrease.

At the same time the contemporary stage of the global tractor-building industry is characterised by emergence of mobile means of energy with high technological properties [5;6]. This provides a possibility to create on their basis such perspective combined machine-and-tractor aggregates, in which one part of the single-operating tool is aggregated from behind and the other one in front of the tractor. Such a scheme of aggregation got the name "push-pull". To implement it, tractors are equipped with a frontal linkage mechanism. However, in practice it can often be observed that ballast weights are used instead of the frontal tool. And the reason for this state of affairs is determined not so much by the lack of frontal machines as by the lack of a scientifically grounded methodology for its aggregation.

The point is that in case the frontal soil tillage machines (especially the energy-intensive ones) are not properly attached, they do not penetrate into the soil. In addition, it can significantly deteriorate (which in practice often happens) the steering ability of the tractor, first of all, of the wheeled one $[5 ; 6]$. The movement stability of the ploughing aggregate, assembled according to the "push-pull" 
scheme in a horizontal plane, can be considered and appropriately evaluated only on condition of stable movement of the buried frontal plough in a longitudinal-vertical plane. Seeking the ways to solve the problem of correct choice of a scheme and parameters of the ploughing aggregates working according to the "push-pull" scheme is the aim of many scientists [3; 7-9]. In their studies they first examined the conditions, under which the frontal plough is buried, the analysis of which made it possible to formulate requirements for the design parameters both of the frontal linkage mechanism of the frontal tool and the frontal linkage mechanism of the tractor.

At the same time these scientists took as an initial assessment criterion the value of the burying moment. But even a superficial analysis shows that a more informative indicator is the magnitude of the reaction upon the supporting wheel of the frontal plough. In the case of the burying moment, the magnitude of this force may be very large, which is extremely undesirable from the energy point of view. An ideal state is one in which the magnitude of the reaction upon the supporting wheel of the frontal plough is slightly greater than zero. Yet, this can only be achieved by correct adjustment of the frontal linkage mechanism of the tractor. As a result, after the scheme and the parameters of the ploughing aggregate, built according to the "push-pull" scheme, are justified, it is necessary to evaluate their impact upon the draft and energy, as well as the operational and technological indicators.

Outside the scope of scientists there remained also issues concerning analysis of the burying conditions of the frontal plough within the ploughing aggregate, built according to the "push-pull" scheme. The more it refers to various installation options of the frontal linking mechanism of the tractor. In addition, for efficient ploughing application of the aggregate, assembled according to the "push-pull" scheme, it is purposeful for the agricultural producers to develop scientifically grounded recommendations on its correct arrangement.

The aim of the work is a theoretical study of the impact of the design parameters of the frontal linkage mechanism of the tractor and the frontal plough upon the movement stability of the ploughing aggregate after it is buried into the soil.

\section{Materials and methods}

In order to solve the set task, we will regard two options of attaching the frontal plough. The first of them is characterised by the fact that the inclination angle $(\alpha)$ of the central drawbar of the frontal linkage mechanism of the tractor is less than the inclination angle $(\beta)$ of the lower links (Option 1: $\alpha<\beta$, Fig. 1). In the second option of aggregation of the considered frontally mounted implement everything is opposite (Option 2: $\alpha>\beta$, Fig. 2).

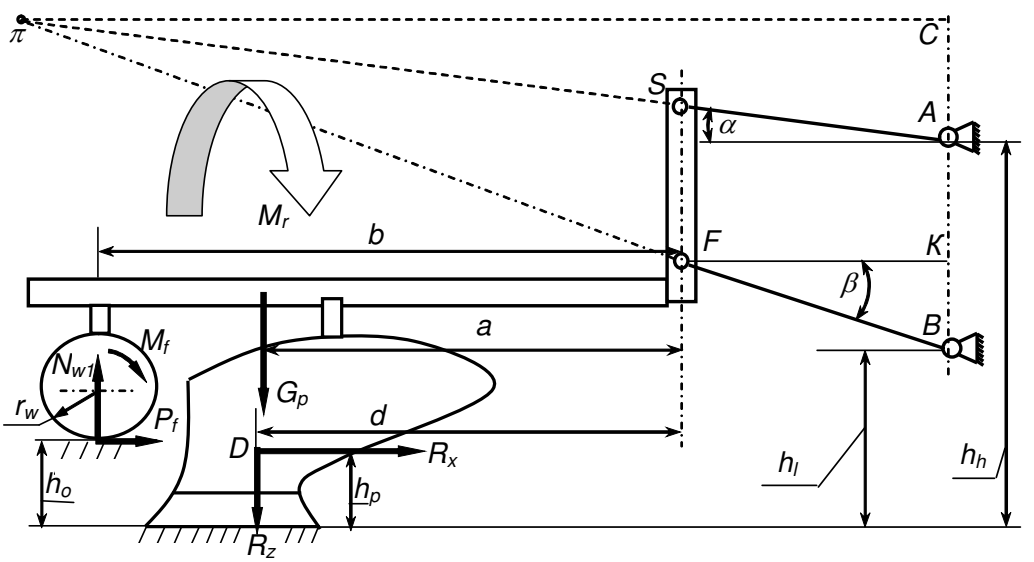

Fig. 1. Diagram of forces acting upon frontal plough with $\alpha<\beta$ (Option 1)

For further analysis we take the following assumptions:

1. We will present the frontal plough as one "equivalent" body. We will concentrate the horizontal $\left(R_{x}\right)$ and the vertical $\left(R_{z}\right)$ components of its draught resistance in the "centre of resistance" - point $D$ (see Fig. 1). 
2. A change in the coordinate position of the supporting wheel of the frontal plough $(b)$ has little impact upon the longitudinal coordinate of its centre of mass ( $a$, Fig. 1).

3. The coordinate of the "resistance centre" of the frontal plough $(d)$ and the longitudinal coordinate of its centre of mass $(a)$ practically coincide (i.e., $a \approx d$ ).

Despite the fact that the forces acting upon the plough in a longitudinal-vertical plane cannot be reduced to a resultant one, the first assumption is widely used by scientists in their studies [4;9], practically without minimising the reliability of the calculations.

The main parts of the mass of the plough are its bodies and the frame. In this respect the movement of the supporting wheel exerts little impact upon the longitudinal coordinate of the centre of mass of the plough, which explains the validity of the second assumption.

The longitudinal coordinate of the "centre of resistance" of the frontal plough is located approximately in the region of the sock point of its second body. As calculations show, practically in the same region there is located the projection of the "centre of mass" of the tool in a horizontal plane. It is this fact that determines the validity of the third assumption.

For Option $1 \alpha<\beta$, (see Fig. 1), after the frontal plough is buried under the action of moment $M_{r}$, the condition of its stable movement in a longitudinal-vertical plane is equality to zero of the sum of the moments acting with respect to the instantaneous turning centre of the frontal linkage mechanism of the tractor (i.e. $\pi$, Fig. 1):

$$
\begin{aligned}
\sum M(\pi)_{1} & =G_{p}\left(\pi C-a-r_{l} \cos \beta\right)+R_{z}\left(\pi C-d-r_{l} \cos \beta\right)-N_{w 1}\left(\pi C-b-r_{l} \cos \beta\right)- \\
& -P_{f}\left(h_{h}+A C-h_{o}\right)-R_{x}\left(h_{h}+A C-h_{p}\right)+M_{f}=0,
\end{aligned}
$$

where $G_{p}$ - force of the weight of the plough, N;

$r_{l}-$ length of the lower links of the frontal linkage mechanism of the tractor, $\mathrm{m}$;

$N_{w 1}$ - vertical reaction on the supporting wheel of the plough, N;

$P_{f}, M_{f}-$ force $(\mathrm{N})$ and torque $(\mathrm{N} \cdot \mathrm{m})$ of the rolling resistance of the wheel of the frontal plough;

$h_{h}$ - coordinate of attachment of the central (upper) drawbar of the frontal linkage mechanism to the tractor, $\mathrm{m}$;

$h_{o}$ - ploughing depth, m;

$h_{l}$ - coordinate of attachment of the lower links of the frontal linkage mechanism of the tractor, m;

$h_{p}$ - vertical coordinate $(\mathrm{m})$ of the "centre of resistance" of the frontal plough - "the equivalent body" - point $D$, Fig. 1;

$\pi C, A C=\pi C \cdot \tan (\alpha)$ - parameters the nature of which is clear from Fig. 1.

From the theory of the tractor and the foundations of agricultural mechanics it is known [4] that:

$$
\left.\begin{array}{l}
P_{f}=\frac{N_{w}}{f_{r}}, \\
M_{f}=\frac{N_{w} r_{w}}{f_{r}}, \\
R_{x}=k_{0} B_{w} h_{o} \\
R_{z}=0.2 R_{x},
\end{array}\right\}
$$

where $N_{w}$ - vertical reaction on the supporting wheel of the plough, $\mathrm{N}$;

$f_{r}$ - coefficient of the rolling resistance;

$r_{w}$ - radius of the supporting wheel of the frontal plough, $\mathrm{m}$;

$k_{0}-$ resistance coefficient of the plough, $\mathrm{N} \cdot \mathrm{m}^{-2}$;

$B_{w}-$ working width of the frontal plough, $\mathrm{m}$.

As it follows from the analysis of Fig. 1, the longitudinal coordinate of the instantaneous turning centre of the frontal linkage mechanism of the tractor $(\pi C)$ can be determined from the following expression: 


$$
\pi C=\frac{h_{h}-h_{l}}{\tan (\beta)-\tan (\alpha)}
$$

Equation (1) describes in a general way the equilibrium state of the frontal plough after it has been buried to the given depth of ploughing. At the same time it should be understood that the above condition will be stable only on condition of a positive response upon the supporting wheel the ploughing implement. That is, when $N_{w 1}>0$.

The impact of all the components of equation (1) upon the value of the force $N_{w 1}$ can be estimated from the dependence that follows directly from (1) taking into account the system of equations (2):

$$
N_{w 1}=\frac{G_{p}\left(\pi C-a-r_{l} \cos \beta\right)+k_{0} B_{w} h_{o}\left[0.2\left(\pi C-d-r_{l} \cos \beta\right)-h_{h}-\pi C \tan (\alpha)+h_{p}\right]}{\left(\pi C-b-r_{l} \cos \beta\right)+\left(h_{h}+\pi C\left[\tan (\alpha)-h_{o}-r_{w}\right]\right) f_{r}^{-1}} .
$$

When justifying the design parameters of the frontal linkage mechanism of the tractor from a position of ensuring stable movement of the frontal implement at a given depth of tillage, an important parameter is the height $(S F)$ of the leg of the connecting triangle of the plough. It follows from Fig. 1, one can determine this value from the following expression:

$$
S F=h_{h}-h_{l}+r_{l}[\cos \beta \cdot \tan (\alpha)-\sin \beta] .
$$

In the option of setting the frontal linkage mechanism of the tractor according to the scheme shown in Fig. 1, the possible range of variation of the inclination angle of the central drawbar should be as follows: $0 \leq \alpha<\beta$. Option $\alpha<0^{\circ}$ is not considered in this study since such an adjustment of the linkage mechanism is characterised by unacceptable kinematics of lifting the frontal implement [6]. The minimum value of the inclination angle of the lower links of the frontal linkage mechanism of the tractor must be greater than $0^{\circ}$. The option $\beta=0^{\circ}$ is not analysed either because, when $\alpha=0^{\circ}$, we obtain a parallelogram mechanism that lifts the implement without its turning in a longitudinally vertical plane (and which is undesirable!). In further analysis we will take into account that in an ideal case the vertical reaction upon the supporting wheel of the frontal plough should be, on the one hand, larger than zero but, on the other hand, close to it. This will be sufficient for a steady movement of the implement along the ploughing depth with minimum waste of energy for rolling its supporting wheel and insignificant deformation of the soil by the latter.

For option $2(\alpha>\beta$, see Fig. 2) the condition of the plough equilibrium in a longitudinally vertical plane is as follows:

$\sum \mathrm{M}(\pi)_{2}=N_{w 2}(\pi C+b)-G_{p}(\pi C+a)-R_{z}(\pi C+d)-P_{f}\left(C \mathrm{E}-h_{o}\right)-R_{x}\left(C E-h_{p}\right)+M_{f}=0$,

where $N_{w 2}-$ vertical reaction on the supporting wheel of the plough, $\mathrm{N}$.

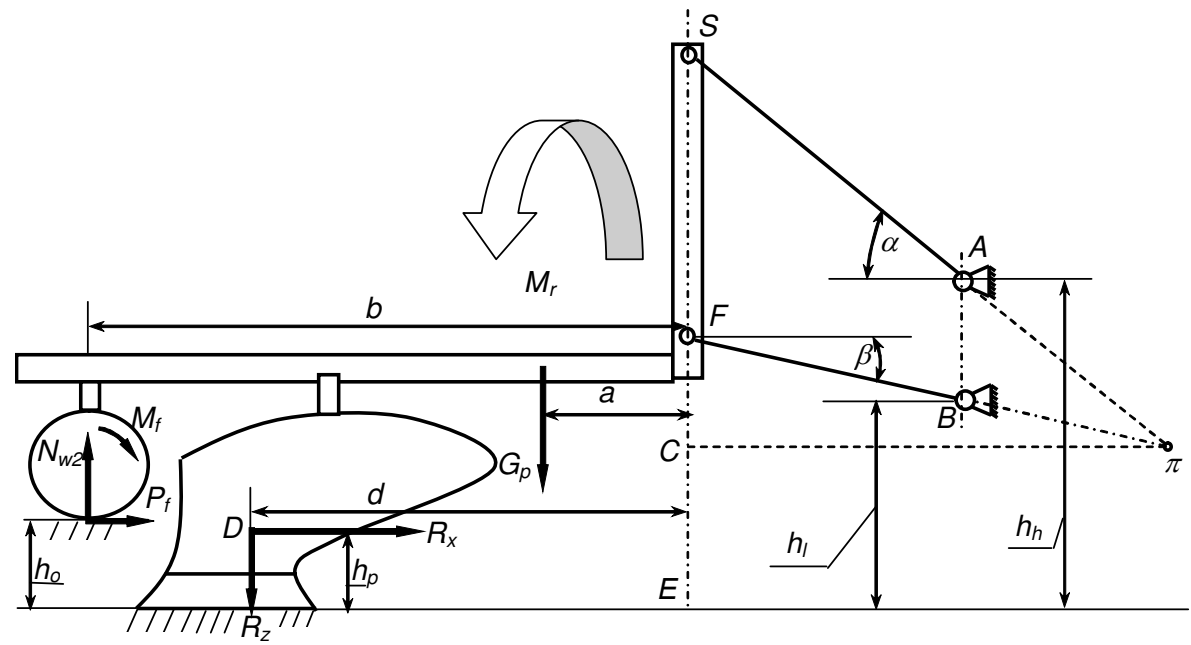

Fig. 2. Diagram of forces acting upon frontal plough at $\alpha>\beta$ (Option 2) 
As it follows from the analysis of Fig. 2, the segment $C E=S E-S C$.

In its turn

$$
\left.\begin{array}{l}
S E=h_{h}+r_{h} \sin \alpha \\
S C=\pi C[\tan (\alpha)],
\end{array}\right\}
$$

where $r_{h}$ - length of the central (upper) link of the frontal linkage mechanism of the tractor, $\mathrm{m}$.

In the justification process of the parameters of the frontal linkage mechanism of the tractor, it is purposeful to express the length of the central drawbar $\left(r_{h}\right)$ as a function of the length of the lower ones $\left(r_{l}\right)$. This is because parameter $r_{l}$ remains constant, when the tractor is aggregated with tools, in contrast to the parameter $r_{h}$. For the adjustment option of the frontally mounted linkage mechanism of the tractor, discussed by us, we have:

$$
r_{h}=\frac{r_{l} \cos \beta}{\cos \alpha} .
$$

Considering dependencies (7) and (8) we obtain:

$$
S E=h_{h}+r_{h} \sin \alpha-\pi C[\tan (\alpha)] .
$$

Substituting expression (9) into (6), after the transformations we obtain:

$N_{w 2}=\frac{G_{p}(\pi C+a)+k_{0} B_{w} h_{o}\left[0.2(\pi C+d)-h_{h}-\left(\pi C-r_{l} \cos \beta\right) \tan (\alpha)-h_{p}\right]}{(\pi C+b)+\left[\left(\pi C-r_{l} \cos \beta\right) \tan (\alpha)+h_{o}+r_{w}-h_{h}\right] f_{r}^{-1}}$.

Besides, in expression (10), the coordinate $\pi C$ is calculated in the following way:

$$
\pi C=\frac{h_{h}-h_{l}+r_{l}[\cos \alpha \cdot \tan (\alpha)-\sin \beta] .}{\tan (\alpha)-\tan (\beta)} .
$$

Dependencies (4) and (10) were calculated from the following values of the design parameters taken for the ploughing aggregate, assembled according to the "push-pull" scheme on the basis of the HTZ-160 tractor [5]: $G_{P}=1-3 \mathrm{kN} ; a=0.55 \mathrm{~m} ; r_{l}=0.82 \mathrm{~m} ; k_{0}=50 \ldots 80 \mathrm{kN} \cdot \mathrm{m}^{-2} ; B_{w}=0.35-1.05 \mathrm{~m}$; $h_{o}=0.22-0.30 \mathrm{~m} ; \quad d=0.4-0.8 \mathrm{~m} ; \quad h_{h}=1.15-1.40 \mathrm{~m} ; \quad h_{l}=0.4-0.8 \mathrm{~m} ; \quad h_{p}=0.5 h_{o} ; \quad b=0.5-1.5 \mathrm{~m} ;$ $r_{w}=0.23 \mathrm{~m} ; f_{r}=0.1$.

\section{Results and discussion}

Taking into consideration the accepted range of variations in angles $\alpha$ and $\beta$, the results of the calculations of expression (4) generally show that, as the inclination angle $\alpha$ of the central drawbar of the frontal linkage mechanism of the tractor increases, the vertical reaction on the supporting wheel of the plough increases, too (Fig. 3).

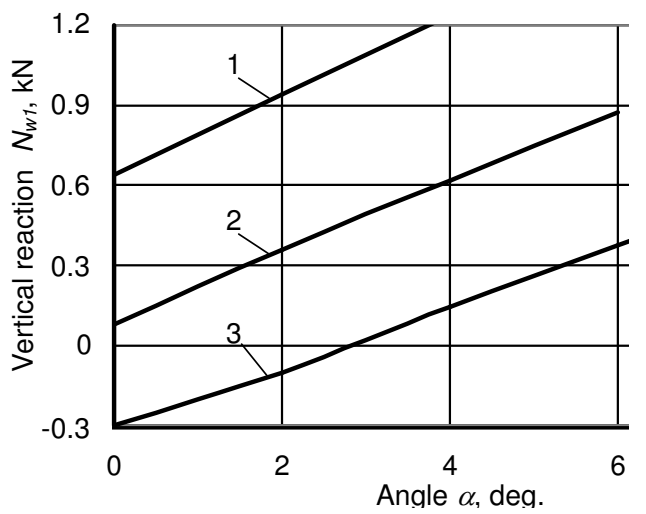

Fig. 3. Dependence of vertical reaction $\left(N_{w 1}\right)$ on supporting wheel of plough on inclination angle of central drawbar $(\alpha)$ at different values of inclination angle of lower links $(\beta)$ of frontal linkage mechanism of tractor: $1-\beta=6^{\circ} ; 2-\beta=8^{\circ} ; 3-\beta=10^{\circ}$ 
The intensity of this process is practically independent of the inclination angle of the lower links $(\beta)$. At the same time the value of this parameter has a rather significant impact upon the magnitude of the force $N_{w 1}$. First, as $\beta$ decreases, it proportionally increases linearly. Second, when the inclination angle of the lower links of the frontal linkage mechanism of the tractor is $10^{\circ}$ and the inclination angle of the central drawbar is within the range of $0 \ldots 3^{\circ}$, the supporting wheel of the plough is completely unloaded. A witness to this is the negative value of the force $N_{w 1}$ (Curve 3, Fig. 3).

It follows from Fig. 3, to adjust the frontal linkage mechanism of the tractor according to the condition $\alpha<\beta$, the selection of the inclination angles of its central drawbar and the lower links should be made considering the following requirements: $0 \leq \alpha<\beta ; 0<\beta \leq 8^{\circ}$.

Let us analyse how the design parameters and the values from expression (4) affect the variation dynamics of the force $N_{w 1}$. It has been established that with an increase in the longitudinal coordinate of the "centre of resistance" of the plough (Point $D$, Fig. 1), the vertical reaction upon the supporting wheel of the plough, although not essentially, but still decreases. Such a result can be explained by the decreased arm of action of the penetrating force $R_{z}$ with the longitudinal coordinate $d$ increasing. Taking into account the small quantitative variations in dependence $N_{w 1}=\mathrm{f}(d)$, the parameter $d$ should be selected as small as possible, since its increase leads to an undesirable growth of the dimensions of the frontal plough. Still less significant impact upon the value of $N_{w 1}$ (within the range of $0.60 \ldots 0.61 \mathrm{kN}$ ) is created by the change in the longitudinal coordinate of the supporting wheel $b$ (see Fig. 1). In quality terms the growth of the parameter $b$ determines an increase in this force. Therefore, from a principal point of view the longitudinal coordinate of the setting of the supporting wheel should be as small as possible. When increasing the ploughing depth $\left(h_{o}\right)$ and the specific resistance $\left(k_{0}\right)$ of the plough, the vertical reaction upon the supporting wheel of the frontal plough decreases. The point is that with the increase of the parameter $h_{o}$, the force $R_{x}$ (which is a lifting force) increases, but the arm of its action decreases, since the "centre of resistance" of the implement rises higher. Since the increase in the magnitude of the force $R_{x}$ outruns the decrease in the arm of its action, the lifting moment increases. As a result, the force $N_{w 1}$ decreases. When the value of $k_{0}$ increases, the longitudinal component of the draught resistance $\left(R_{x}\right)$ of the plough also increases. But since the burying (penetration) moment increases, the vertical reaction upon the supporting wheel of the plough correspondingly decreases. The other thing is that in quantitative terms the impact of the change in the parameters $h_{o}$ and $k_{0}$ is unimportant and can be neglected in practical calculations. This result is very important, since it determines the agrotechnical requirements for the depth of ploughing. Now let us analyse how the vertical coordinates of attachment of the central $\left(h_{h}\right)$ drawbar and the lower $\left(h_{1}\right)$ links of the frontal linkage mechanism of the tractor affect the change in the force $N_{w 1}$. It has been established by the calculations of dependence (4) that in case the parameter $h_{1}$ is increased two times (from 0.4 to $0.8 \mathrm{~m}$ ), the value of the vertical reaction on the supporting wheel of the plough decreases practically as many times (Fig. 4 ).

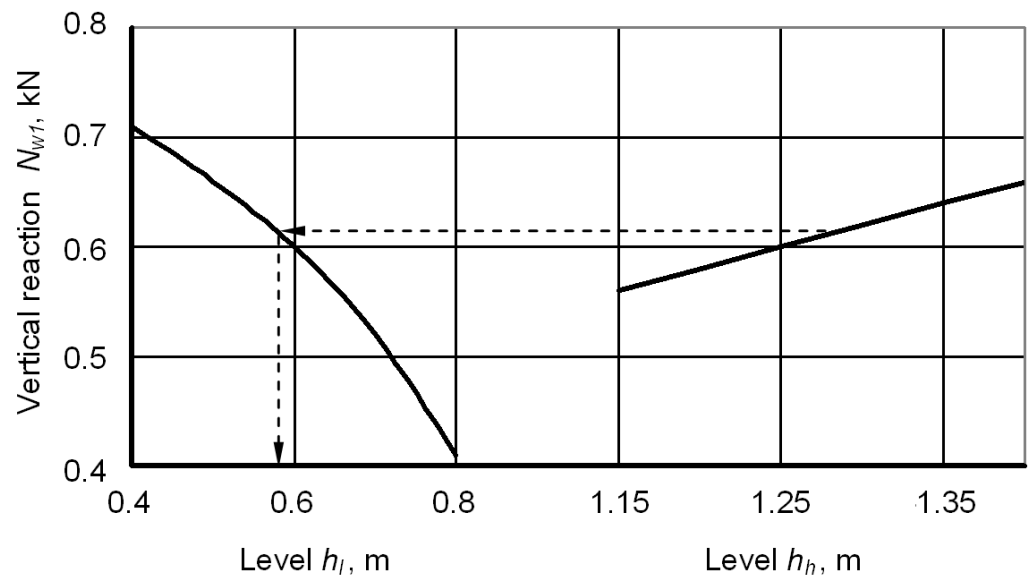

Fig. 4. Dependence of vertical reaction $\left(N_{w 1}\right)$ upon supporting wheel of plough on height of attachment of lower $\left(h_{l}\right)$ links and central $\left(h_{h}\right)$ drawbar of frontal linkage mechanism of tractor

At the same time, when the height of the central drawbar of the frontal linkage mechanism of the aggregating tractor increases, we have an opposite result, although in this case the process is 
characterised by a much lower intensity. It is much easier in practice to change the coordinate $h_{1}$ than coordinate $h_{h}$ of the frontal linkage mechanism of the tractor. In this connection, in our case the attachment height of the central drawbar to the tractor is assumed to be the same as that of the serial tractor of the HTZ-160 family, namely: $h_{h}=1.27 \mathrm{~m}$. Seeking a compromise solution between the two alternative options by parameters $h_{h}$ and $h_{1}$ should take place according to the following algorithm:

1. from the dependence $N_{w 1}=f\left(h_{h}\right)$ we determine the vertical reaction upon the wheel of the frontal plough according to the accepted value of the setting coordinate of the central drawbar of the frontal linkage mechanism of the tractor;

2. from the value of the force $N_{w 1}$ obtained from the graphical dependence $N_{w 1}=\mathrm{f}\left(h_{l}\right)$ we determine the vertical setting coordinate of the lower links of the frontal linkage mechanism of the aggregating tractor;

3. for the selected values of $h_{h}$ and $h_{1}$ and the adopted inclination angles $\alpha$ and $\beta$ of the frontal linkage mechanism of the tractor we determine from dependence (5) the height of the leg SF of the coupling triangle of the frontally mounted plough.

In this case for a two-bottom frontal plough at $\alpha=0^{\circ} ; \beta=8^{\circ} ; G_{p}=2 \mathrm{kN} ; a=0.55 \mathrm{~m} ; r_{l}=0.82 \mathrm{~m}$; $k_{0}=65 \mathrm{kN} \cdot \mathrm{m}^{-2} ; \quad B_{w}=0.70 \mathrm{~m} ; h_{o}=0.26 \mathrm{~m} ; d=0.6 \mathrm{~m} ; b=1.0 \mathrm{~m} ; r_{w}=0.23 \mathrm{~m} ; f_{r}=0.1$ we have: $h_{h}=1.27 \mathrm{~m} ; h_{l}=0.58 \mathrm{~m} ; S F=0.58 \mathrm{~m}$. The vertical reaction on the supporting wheel of the frontal plough is $0.2 \mathrm{kN}$, which is quite acceptable. In option $2(\alpha>\beta$, Fig. 2$)$ of setting the frontal linkage mechanism of the tractor the increase in the inclination angle of its central drawbar also leads to the increase in the vertical reaction $\left(N_{w 2}\right)$ on the supporting wheel of the frontal plough, though with less intensity (Fig. 5).

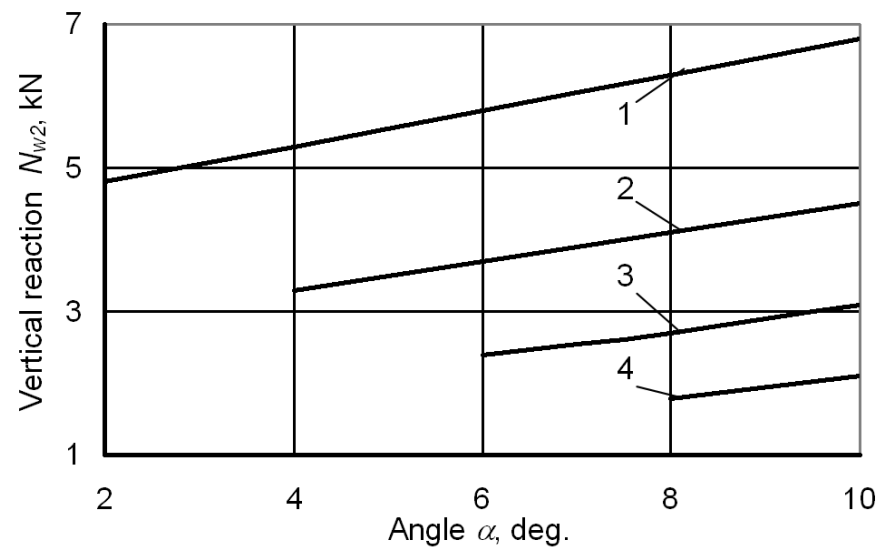

Fig. 5. Dependence of vertical reaction $\left(N_{w 2}\right)$ upon supporting wheel of plough on inclination angle $(\alpha)$ of central drawbar at different values of inclination angle $(\beta)$ of lower links of frontal linkage mechanism of aggregating tractor: $1-\beta=0^{\circ} ; 2-\beta=2^{\circ}$;

$$
3-\beta=4^{\circ} ; 4-\beta=6^{\circ}
$$

The process of changing the dynamics of the vertical reaction upon the supporting wheel of the frontal plough witnesses that the maximum value of the force $N_{w 2}$ is $6.85 \mathrm{kN}$, which is 5.5 times more than in comparison with option 1 (see Fig. 3). Of course, the maximum values of the reaction $N_{w 2}$ are achieved in this case at various inclination angles of the central drawbar and the lower links of the frontal linkage mechanism of the tractor. The nature of the impact of other design and technological parameters of the frontal plough upon the magnitude of the vertical additional load of its supporting wheel is the following. As it follows from the analysis of the calculation of expression (10), if the ploughing depth $\left(h_{o}\right)$ and specific resistance $\left(k_{0}\right)$ of the ploughing implement increases, force $N_{w 2}$ grows, although insignificantly. Such a result is logical since, in this scheme of setting the frontal linkage mechanism of the tractor, the force $R_{x}$ is burying (penetrating) (see Fig. 2), and its value depends, as it follows from (2), on the product of the values $h_{o}$ and $k_{0}$. In comparison with the variant of setting the frontal linkage mechanism of the tractor according to option $1(\alpha<\beta)$, setting the central drawbar at a larger angle than the lower links (Option 2, $\alpha>\beta$ ) shows that the nature of dependencies $N_{w 2}=\mathrm{f}(b ; d)$ is the opposite. And again, in quantitative terms the impact of the design parameters $b$ and $d$ is negligible; therefore recommendations for their selection are the same as in the variant for 
setting the frontal linkage mechanism of the tractor for option 1. In comparison with option $1(\alpha<\beta)$, opposite is also the nature of the variations in the vertical load $\left(N_{w 2}\right)$ upon the plough wheel, depending on the vertical setting coordinates of the central drawbar $\left(h_{h}\right)$ and the lower links $\left(h_{1}\right)$ of the frontal linkage mechanism of the tractor (Fig. 6). The determination algorithm of rational values of these coordinates is assumed to be the same as in the previous option 1 of setting the frontal linkage mechanism of the tractor; namely, assuming that $h_{h}=1.27 \mathrm{~m}$, from Fig. 6 we find the coordinate $h_{1}$, equal to $0.59 \mathrm{~m}$. Finally, we will determine how the magnitude of the vertical reaction on the supporting wheel of the frontal plough depends on its working width $\left(B_{w}\right)$ for various setting schemes of the frontal linkage mechanism of the tractor.

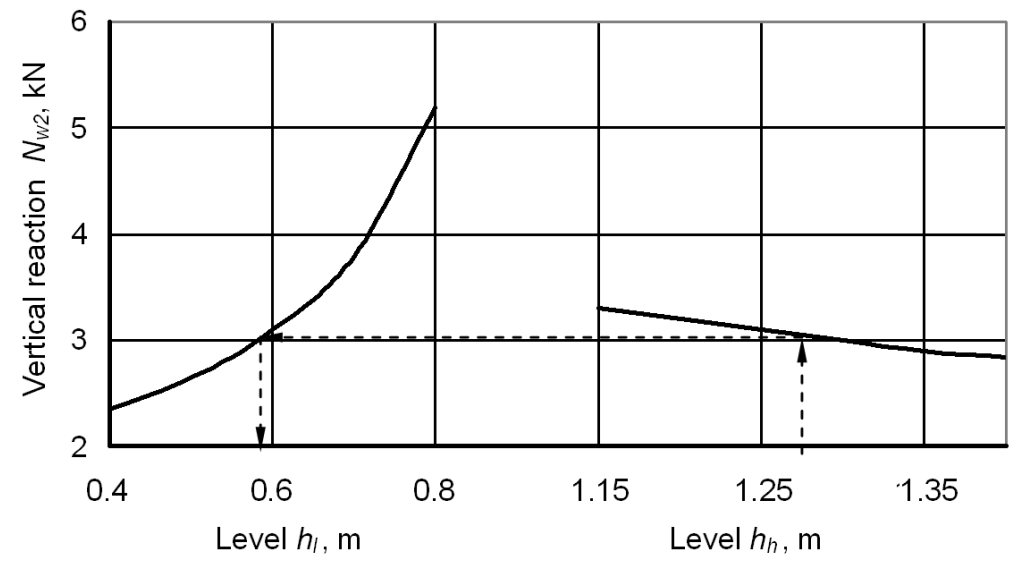

Fig. 6. Dependence of vertical reaction $\left(N_{w 2}\right)$ on supporting wheel of plough upon attachment height of lower links $\left(h_{1}\right)$ and central drawbar $\left(h_{h}\right)$ of frontal linkage mechanism to tractor

Calculations show that the greatest impact is exerted by option 2, when the inclination angle of the central drawbar is greater than the installation angle of the lower links of the frontal linkage mechanism of the tractor (Fig. 7).

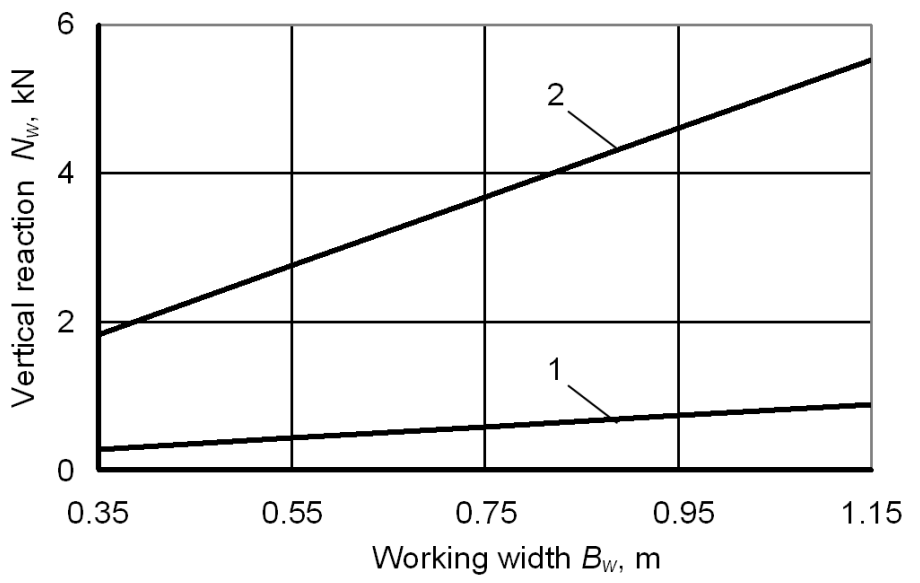

Fig. 7. Dependence of vertical reaction $\left(N_{w}\right)$ on supporting wheel of frontal plough upon working width $\left(B_{w}\right)$ at various settings of frontal linkage mechanism of tractor:

$$
1 \text { - Option } 1 \alpha<\beta) ; 2 \text { - Option } 2(\alpha>\beta)
$$

The difference in the loading of the wheel of a ploughing implement is particularly noticeable, when the value of $B_{w}$ increases. Thus, if using a single-bottom frontal plough it is $1.8 \mathrm{kN}$, then in the variant of a three-bottom implement this value rises to $5.5 \mathrm{kN}$, that is, more than three times.

The achieved result should be obligatory taken into account, when analysing the dynamics of the movement of the ploughing aggregate according to the "push-pull" scheme in a horizontal plane. The point is that in case there is increased vertical load upon the wheel of the frontal plough, the force of friction between this wheel and the rolling surface (soil) increases. In turn, if there is a control impact of the turning angle of the driven wheels of the tractor, the latter tries to shift the frontally mounted implement in a transverse direction. And in a certain way this shift will depend precisely on the 
vertical load, with which the supporting wheel of the ploughing implement is pressed against the surface of the field. In any case, the lower the load, the less force is needed for transverse movement of the frontal plough.

\section{Conclusions}

1. As a result of theoretical studies it has been established that, to ensure the stability of the movement of a buried frontal plough within the ploughing aggregate, assembled according to the "push-pull" scheme, the inclination angle of the central drawbar $(\alpha)$ of the frontal linkage mechanism of the tractor should be less than the inclination angle of its lower links $(\beta)$. To adjust the frontal linkage mechanism of the tractor according to condition $\alpha<\beta$, the selection of these angles should be made taking into account the following requirements: $0 \leq \alpha<\beta ; 0<\beta \leq 8^{\circ}$.

2. Considering the insignificant impact on the vertical additional loading of the frontal plough and the significant impact upon its dimensions, the longitudinal coordinates of the "centre of resistance" and the setting point of the supporting wheel of the ploughing implement attached to the tractor at the inclination angle of the central drawbar less than the inclination angle of the lower links, should be as small as possible.

3. When the frontal plough is pivotally attached to the frontal linkage mechanism of the tractor with the inclination angle of the central drawbar larger than the inclination angle of the lower links, the vertical load on the supporting wheel of the ploughing implement is greater. In contrast to the setting variant of the frontal linkage mechanism of the tractor, when the inclination angle of the central drawbar is less than the inclination angle of the lower links, the vertical load on the supporting wheel of the ploughing implement is 5.5 times larger. Besides, the maximum values of the reaction on the supporting wheel are achieved at various inclination angles of the central drawbar and the lower links of the frontal linkage mechanism of the tractor.

4. Comparative analysis of the attachment schemes of the frontal plough shows that in the attachment variant of the frontal plough to the frontal linkage mechanism of the aggregating tractor with the inclination angle of the central drawbar greater than the inclination angle of the lower links, the vertical load upon its supporting wheel is three times less than in the variant, when the inclination angle of the central drawbar is less than the inclination angle of the lower links.

\section{References}

[1] Vilde A., Rucins A. Simulation of the Impact of the Plough Body Parameters, Soil Properties and Working Modes on the Ploughing Resistance. $10^{\text {th }}$ International Conference on Computer Modelling and Simulation. Emmanuel College Cambridge, 1 - 3 April 2008, pp. 697-702.

[2] Barwicki J., Gach S., Ivanovs S. Proper utilization of soil structure for crops today and conservation for future. Engineering for Rural Development, Vol. 11, 2012, pp. 10-15.

[3] Köller K. Frontpluge. Agrar-übersticht 1, 1983, pp. 12-13 (In German)

[4] Панченко А., Кюрчев В. Исследование тягово-сцепных качеств колесных тракторов (А study of the draft and coupling qualities of wheeled tractors), Труды Таврического государственного агротехнологического университета (Proceedings of the Taurida State Agrotechnological University), Issue 8, vol. 9, Melitopol, 2008, pp. 31-36. (In Ukrainian)

[5] Kyurchev V. Technical change and financial efficiency: plowing-rotary tractors in Ukraine. Journal of Finance and Accounting, No 2 (1), 2014, pp. 8-12

[6] Kyurchev V. Prospects for the use of combined machine-and-tractor aggregate. Science news, Vol. XXI, No. 5, Varna, 2013, pp. 104-108

[7] Li Z., Mitsuoka M., Inoue E., Okayasu T., Hirai Y., Zhu Z. Modification of a tractor dynamic model considering the rotatable front end. Journal of the Faculty of Agriculture, Vol. 60(1), 2015, pp. 219-224.

[8] Bulgakov V., Adamchuk V., Ivanovs S., Ihnatiev Y. Theoretical investigation of aggregation of top removal machine frontally mounted on wheeled tractor. Engineering for rural development, Vol. 16, 2017. p. 273-280

[9] Nadykto V., Kyurchev V., Beloev H., Kistechok A. Study of push-pull plough combination. Journal of agriculture and environment. Vol.1, No 1, 2017, pp. 4-9. 